Kragujevac Journal of Mathematics

Volume 45(1) (2021), Pages 139-154.

\title{
THE RECIPROCAL COMPLEMENTARY WIENER NUMBER OF GRAPH OPERATIONS
}

\author{
R. NASIRI, A. NAKHAEI, AND A. R. SHOJAEIFARD
}

\begin{abstract}
The reciprocal complementary Wiener number of a connected graph $G$ is defined as $\sum_{\{x, y\} \subseteq V(G)} \frac{1}{D+1-d_{G}(x, y)}$, where $D$ is the diameter of $G$ and $d_{G}(x, y)$ is the distance between vertices $x$ and $y$. In this work, we study the reciprocal complementary Wiener number of various graph operations such as join, Cartesian product, composition, strong product, disjunction, symmetric difference, corona product, splice and link of graphs.
\end{abstract}

\section{INTRODUCTION}

Throughout this work, all graphs considered are simple, connected and finite. Let $G=(V(G), E(G))$ be a connected graph. For $x, y \in V(G)$, the distance $d_{G}(x, y)$ between the vertices $x$ and $y$ is equal to the length of a shortest path that connects $x$ and $y$. For a vertex $x$ in a connected nontrivial graph $G$, the eccentricity $\varepsilon_{G}(x)$ of $x$ is the greatest geodesic distance between $x$ and any other vertex of $G$. Also, the diameter $D=D(G)$ of the graph $G$ is defined as the maximum eccentricity of any vertex in $G$. In other words,

$$
\varepsilon_{G}(x)=\max \left\{d_{G}(x, y) \mid y \in V(G)\right\}, \quad D=D(G)=\max \left\{\varepsilon_{G}(x) \mid x \in V(G)\right\} .
$$

In mathematical chemistry, a molecular graph (or chemical graph) is a labeled graph whose vertices correspond to the atoms of the compound and edges correspond to chemical bonds. It is natural to study mathematical properties of these graph models to find chemico-physical properties of the molecule under consideration.

Let $G$ be a $n$-vertex graph with the vertex-set $V(G)=\left\{v_{1}, v_{2}, \ldots, v_{n}\right\}$ and diameter $D$. The reciprocal complementary distance matrix $R C D=\left[r c_{i j}\right]$ of $G$ is an $n \times n$

Key words and phrases. Reciprocal complementary Wiener number, distance, graph operations. 2010 Mathematics Subject Classification. 05C12, 05C35.

DOI 10.46793/KgJMat2101.139N

Received: February 04, 2018.

Accepted: October 27, 2018. 
matrix such that $r c_{i j}=\frac{1}{D+1-d_{G}\left(v_{i}, v_{j}\right)}$ if $i \neq j$, and 0 otherwise (see [7]). Ivanciuc et al. $[5,6]$ introduced the reciprocal complementary Wiener number of the graph $G$ as:

$$
R C W(G)=\sum_{i=1}^{n-1} \sum_{j=i+1}^{n} r c_{i j}=\sum_{\left\{v_{i}, v_{j}\right\} \subseteq V(G)} \frac{1}{D+1-d_{G}\left(v_{i}, v_{j}\right)} .
$$

This invariant has been successfully applied in the structure-property modeling of the molar hear capacity, standard Gibbs energy of formation and vaporization enthalpy of 134 alkanes $C_{6} C_{10}$ (see [5]).

Zhou et al. [14] gave various bounds for this quantity and Nordhaus-Gaddumtype result. Moreover, the trees with the smallest, the second smallest and the third smallest $R C W$, and the unicyclic and bicyclic graphs with the smallest and the second smallest $R C W$ are characterized (see [2]). Zhu et al. [15] obtained the unique tree with $4 \leq D \leq n-3$ and minimum reciprocal complementary Wiener number. They also specified the non-caterpillars with the smallest, the second smallest and the third smallest $R C W$-value. In [10], some bounds for the reciprocal complementary Wiener index of line graphs are presented.

Up to now, various topological indices have been introduced and used in the QSAR/QSPR studies. The Wiener index (or Wiener number) is the oldest and is one of the most studied topological quantities, both from a theoretical point of view and applications. This concept is defined as the sum of distances over all unordered vertex pairs in a graph $G$ (see [12]). This invariant obtained wide attention and numerous results have been worked out, see the survey [13]. In special classes of graphs, such as trees, unicyclic and bicyclic graphs, this index has been studied in $[3,9,11]$. After it, a large number of other distance-based topological indices have been proposed and considered in the chemical and mathematico-chemical literature.

Brückler et al. [1] introduced a general distance-based topological index, called $Q$-index. The $Q$-index is defined as

$$
Q(G)=\sum_{k \geq 0} f(k) D(G, k)
$$

where $f$ is a function such that $f(0)=0$, and $D(G, k)$ is the number of vertex pairs at distance $k . Q$ is an additive function of increments associated with pairs of vertices of $G$. The Wiener, hyper-Wiener, Harary, and reciprocal complementary Wiener indices are all special cases of the $Q$-index. More precisely, by choosing $f(k)=k, \frac{k^{2}}{2}+\frac{k}{2}, \frac{1}{k}$ and $\frac{k^{3}}{6}+\frac{k^{2}}{2}+\frac{k}{3}$, the $Q$-index is equal to the Wiener, hyperWiener, Harary, and Tratch-Stankevich-Zefirov indices, respectively. In other special case, if consider $f(k)=\frac{1}{D+1-k}$, then the $Q$-index will be equal to the reciprocal complementary Wiener number. In other words, it holds

$$
R C W(G)=\sum_{k=1}^{D} \frac{D(G, k)}{D+1-k}
$$


In this research, we study the reciprocal complementary Wiener number of various graph operations like join, Cartesian product, composition, strong product, disjunction, symmetric difference, corona product, splice and link of graphs.

\section{Main Results}

Throughout this paper, we consider graphs $G_{i}$ with $n_{i}$ vertices, $m_{i}$ edges and the diameter $D_{i}, i=1,2$. Also, note that whenever we say $x y \notin E$, it is assumed that $x \neq y$. Moreover, we use standard notations of graph theory. The path, cycle, star, wheel and complete graphs with $n$ vertices are denoted by $P_{n}, C_{n}, S_{n}, W_{n}$ and $K_{n}$, respectively.

By applying relation (1.3), we compute $R C W$ of some special graphs in the following example.

Example 2.1. Let $P_{n}, K_{n}, S_{n}$ and $W_{n}$ denote a path graph, complete graph, star graph and wheel graph with $n$ vertices, respectively. Then

$$
\begin{aligned}
R C W\left(P_{n}\right) & =n-1, \\
R C W\left(K_{n}\right) & =\frac{1}{2} n(n-1), \\
\operatorname{RCW}\left(S_{n}\right) & =\frac{1}{2}(n-1)^{2}, \\
\operatorname{RCW}\left(W_{n}\right) & = \begin{cases}6, & n=4, \\
\frac{1}{2}(n-1)(n-2), & n \geq 5 .\end{cases}
\end{aligned}
$$

We begin by computing the reciprocal complementary Wiener number of join of graphs.

2.1. Join. The join $G_{1}+G_{2}$ of graphs $G_{1}$ and $G_{2}$ with disjoint vertex sets $V_{1}$ and $V_{2}$ is the graph union $G_{1} \cup G_{2}$ together with all the edges joining $V_{1}$ and $V_{2}$. In the following lemma, we determine the reciprocal complementary Wiener number of join of graphs with respect to their numbers of vertices and edges.

Theorem 2.1. Let $G_{1}$ and $G_{2}$ be two $n_{1}$ - and $n_{2}$-vertex graphs, respectively.

(i) If $G_{1}$ and $G_{2}$ are complete graphs, then

$$
R C W\left(G_{1}+G_{2}\right)=\frac{1}{2}\left(n_{1}+n_{2}\right)\left(n_{1}+n_{2}-1\right) .
$$

(ii) If $\left\{G_{1}, G_{2}\right\} \neq\left\{K_{n_{1}}, K_{n_{2}}\right\}$, then

$$
R C W\left(G_{1}+G_{2}\right)=\frac{1}{2}\left(n_{1}\left(n_{1}+n_{2}-1\right)+n_{2}\left(n_{2}-1\right)-m_{1}-m_{2}\right) .
$$


Proof. Suppose $x$ and $y$ are two vertices of $G_{1}+G_{2}$. By definition of the join of two graphs, one can easily see that

$$
d_{G_{1}+G_{2}}(x, y)= \begin{cases}0, & x=y, \\ 1, & x y \in E_{1} \text { or } x y \in E_{2} \text { or }\left(x \in V_{1} \text { and } y \in V_{2}\right), \\ 2, & \text { otherwise. }\end{cases}
$$

Assume that $G_{1}$ and $G_{2}$ are complete graphs, then $G_{1}+G_{2}=K_{n_{1}+n_{2}}$. Therefore, $R C W\left(G_{1}+G_{2}\right)=R C W\left(K_{n_{1}+n_{2}}\right)=\left(\begin{array}{c}n_{1}+n_{2} \\ 2\end{array}\right)$ (see Example 2.1). This completes the proof of part (i). To prove the second part, suppose that at least one of graphs $G_{1}$ or $G_{2}$ is not complete. So, we have $D=D\left(G_{1}+G_{2}\right)=2$, and

$$
\begin{aligned}
R C W\left(G_{1}+G_{2}\right)= & \sum_{\{x, y\} \subseteq V\left(G_{1}+G_{2}\right)} \frac{1}{3-d_{G_{1}+G_{2}}(x, y)} \\
= & \sum_{\{x, y\} \subseteq V_{1}} \frac{1}{3-d_{G_{1}+G_{2}}(x, y)}+\sum_{\{x, y\} \subseteq V_{2}} \frac{1}{3-d_{G_{1}+G_{2}}(x, y)} \\
& +\sum_{\substack{x \in V_{1} \\
y \in V_{2}}} \frac{1}{3-d_{G_{1}+G_{2}}(x, y)} \\
= & \sum_{x y \in E_{1}} \frac{1}{3-d_{G_{1}+G_{2}}(x, y)}+\sum_{x y \notin E_{1}} \frac{1}{3-d_{G_{1}+G_{2}}(x, y)} \\
& +\sum_{x y \in E_{2}} \frac{1}{3-d_{G_{1}+G_{2}}(x, y)}+\sum_{x y \notin E_{2}} \frac{1}{3-d_{G_{1}+G_{2}}(x, y)} \\
& +\sum_{\substack{x \in V_{1} \\
y \in V_{2}}} \frac{1}{3-d_{G_{1}+G_{2}}(x, y)} \\
= & \frac{1}{2}\left(n_{1}\left(n_{1}+n_{2}-1\right)+n_{2}\left(n_{2}-1\right)-m_{1}-m_{2}\right) .
\end{aligned}
$$

Example 2.2. We know that $\overline{K_{r}}+\overline{K_{s}}=K_{r, s}$ (in particular, $K_{1}+\overline{K_{n-1}}=K_{1, n-1}=S_{n}$ ) is the complete bipartite graph. From Theorem 2.1 we obtain explicit formulas for the reciprocal complementary Wiener number of the these graphs

$$
R C W\left(K_{r, s}\right)=\frac{1}{2}(r(r+s-1)+s(s-1)), \quad R C W\left(S_{n}\right)=\frac{1}{2}(n-1)^{2} .
$$

2.2. Cartesian product. The Cartesian product $G_{1} \square G_{2}$ of graphs $G_{1}$ and $G_{2}$ has the vertex set $V_{1} \times V_{2}$ and $(x, y)(u, v)$ is an edge of $G_{1} \square G_{2}$ if ( $x=u$ and $y v \in E_{2}$ ), or $\left(x u \in E_{1}\right.$ and $\left.y=v\right)$. For example, the ladder graph $L_{2, n}$ can be obtained as the Cartesian product of two path graphs $P_{2}$ and $P_{n}$.

Now, we study the reciprocal complementary Wiener number of the Cartesian product of graphs. To do this, we need the following well-known relation related to distance properties of the Cartesian product of two graphs (see [4])

$$
d_{G_{1} \square G_{2}}((x, y),(u, v))=d_{G_{1}}(x, u)+d_{G_{2}}(y, v) .
$$


Theorem 2.2. Let $G_{1}$ and $G_{2}$ be two non-complete graphs. Then

$$
\begin{aligned}
R C W\left(G_{1} \square G_{2}\right)< & \frac{n_{1} m_{2}+n_{2} m_{1}}{D_{1}+D_{2}}+\frac{2 m_{1} m_{2}}{D_{1}+D_{2}-1}+\left(n_{1}+2 m_{1}\right)\left(R C W\left(G_{2}\right)-\frac{m_{2}}{D_{2}}\right) \\
& +\left(n_{2}+4 m_{2}\right)\left(R C W\left(G_{1}\right)-\frac{m_{1}}{D_{1}}\right) .
\end{aligned}
$$

Proof. Applying (2.1), we have $D=D\left(G_{1} \square G_{2}\right)=D_{1}+D_{2}$. Therefore,

$$
\begin{aligned}
& R C W\left(G_{1} \square G_{2}\right)=\sum_{\{(x, y),(u, v)\} \subseteq V\left(G_{1} \square G_{2}\right)} \frac{1}{D+1-d_{G_{1} \square G_{2}}((x, y),(u, v))} \\
& =\sum_{\substack{\{(x, y),(x, v)\} \subseteq V\left(G_{1} \square G_{2}\right) \\
y v \in E_{2}}} \frac{1}{D_{1}+D_{2}} \\
& +\sum_{\{(x, y),(x, v)\} \subseteq V\left(G_{1} \square G_{2}\right)} \frac{1}{D_{1}+D_{2}+1-d_{G_{2}}(y, v)} \\
& +\sum_{\substack{\{(x, y),(u, y)\} \subseteq V\left(G_{1} \square G_{2}\right) \\
x u \in E_{1}}} \frac{1}{D_{1}+D_{2}} \\
& +\sum_{\{(x, y),(u, y)\} \subseteq V\left(G_{1} \square G_{2}\right)}^{x u \notin E_{1}} \frac{1}{D_{1}+D_{2}+1-d_{G_{1}}(x, u)} \\
& +\sum_{\substack{\{(x, y),(u, v)\} \subseteq V\left(G_{1} \square G_{2}\right) \\
x u \in E_{1}, y v \in E_{2}}} \frac{1}{D_{1}+D_{2}-1} \\
& +\sum_{\substack{\{(x, y),(u, v)\} \subseteq V\left(G_{1} \square G_{2}\right) \\
x u \in E_{1}, y v \notin E_{2}}} \frac{1}{D_{1}+D_{2}-d_{G_{2}}(y, v)} \\
& +\sum_{\substack{\{(x, y),(u, v)\} \subseteq V\left(G_{1} \square G_{2}\right) \\
x u \notin E_{1}, y v \in E_{2}}} \frac{1}{D_{1}+D_{2}-d_{G_{1}}(x, u)} \\
& +\sum_{\substack{\{(x, y),(u, v)\} \subseteq V\left(G_{1} \square G_{2}\right) \\
x u \notin E_{1}, y v \notin E_{2}}} \frac{1}{D_{1}+D_{2}+1-d_{G_{1}}(x, u)-d_{G_{2}}(y, v)} \\
& <\frac{n_{1} m_{2}}{D_{1}+D_{2}}+n_{1}\left(R C W\left(G_{2}\right)-\frac{m_{2}}{D_{2}}\right)+\frac{n_{2} m_{1}}{D_{1}+D_{2}} \\
& +n_{2}\left(R C W\left(G_{1}\right)-\frac{m_{1}}{D_{1}}\right)+\frac{2 m_{1} m_{2}}{D_{1}+D_{2}-1} \\
& +2 m_{1}\left(R C W\left(G_{2}\right)-\frac{m_{2}}{D_{2}}\right)+2 m_{2}\left(R C W\left(G_{1}\right)-\frac{m_{1}}{D_{1}}\right) \\
& +2 m_{2}\left(R C W\left(G_{1}\right)-\frac{m_{1}}{D_{1}}\right)
\end{aligned}
$$




$$
\begin{aligned}
= & \frac{n_{1} m_{2}+n_{2} m_{1}}{D_{1}+D_{2}}+\frac{2 m_{1} m_{2}}{D_{1}+D_{2}-1}+\left(n_{1}+2 m_{1}\right)\left(R C W\left(G_{2}\right)-\frac{m_{2}}{D_{2}}\right) \\
& +\left(n_{2}+4 m_{2}\right)\left(R C W\left(G_{1}\right)-\frac{m_{1}}{D_{1}}\right) .
\end{aligned}
$$

Corollary 2.1. Let $G_{1} \cong K_{n_{1}}$ and $G_{2} ¥ K_{n_{2}}$ be two graphs. Then

$$
\begin{aligned}
R C W\left(G_{1} \square G_{2}\right)= & \frac{n_{1} m_{2}+n_{2}\left(\begin{array}{c}
n_{1} \\
2
\end{array}\right)}{1+D_{2}}+\frac{2\left(\begin{array}{c}
n_{1} \\
2
\end{array}\right) m_{2}}{D_{2}}+2\left(\begin{array}{c}
n_{1} \\
2
\end{array}\right)\left(R C W\left(G_{2}\right)-\frac{m_{2}}{D_{2}}\right) \\
& +\sum_{y v \notin E_{2}} \frac{n_{1}}{D_{2}+2-d_{G_{2}}(y, v)} \\
< & \frac{n_{1} m_{2}+n_{2}\left(\begin{array}{c}
n_{1} \\
2
\end{array}\right)}{1+D_{2}}+\frac{2\left(\begin{array}{c}
n_{1} \\
2
\end{array}\right) m_{2}}{D_{2}}+\left(n_{1}+2\left(\begin{array}{c}
n_{1} \\
2
\end{array}\right)\right)\left(R C W\left(G_{2}\right)-\frac{m_{2}}{D_{2}}\right) .
\end{aligned}
$$

Example 2.3. Consider the graph whose vertices are the $n$-tuples $b_{1}, b_{2}, \ldots, b_{n}$ with $b_{i} \in$ $\{0,1\}$, let two vertices be adjacent if the corresponding tuples differ in precisely one place. Such a graph is called a hypercube of dimension $n$ and denoted by $Q_{n}$. It is wellknown fact that the hypercube $Q_{n}$ can be written in the form $Q_{n}=\underbrace{K_{2} \square K_{2} \square \cdots \square K_{2}}_{n \text { times }}$.

For $n=3$, by Corollary 2.1 we have

$$
R C W\left(Q_{3}\right)=K_{2} \square K_{2} \square K_{2}=K_{2} \square C_{4}=14 .
$$

Corollary 2.2. Let $L_{2, n}$ be the ladder graph, then

$$
R C W\left(L_{2, n}\right)=4 n-1-2 \sum_{k=1}^{n} \frac{1}{k} .
$$

Proof.

$$
\begin{aligned}
R C W\left(L_{2, n}\right) & =R C W\left(P_{2} \square P_{n}\right) \\
& =\frac{2(n-1)+n}{n}+\frac{2(n-1)}{n-1}+2(n-2)+2 \sum_{y v \notin E\left(P_{n}\right)} \frac{1}{n+1-d_{P_{n}}(y, v)} \\
& =-\frac{2}{n}+2 n+1+2 \sum_{k=2}^{n-1} \frac{D\left(P_{n}, k\right)}{n+1-k} .
\end{aligned}
$$

On the other hand, it is clear that $D\left(P_{n}, k\right)=n-k$, for $k=1, \ldots, n-1$. Therefore,

$$
\begin{aligned}
R C W\left(L_{2, n}\right) & =-\frac{2}{n}+2 n+1+2 \sum_{k=2}^{n-1} \frac{n-k}{n+1-k} \\
& =4 n-1-2 \sum_{k=1}^{n} \frac{1}{k} .
\end{aligned}
$$

Corollary 2.3. Let $G_{1} \cong K_{n_{1}}$ and $G_{2} \cong K_{n_{2}}$ be two complete graphs. Then

$$
R C W\left(G_{1} \square G_{2}\right)=\frac{n_{1} n_{2}}{4}\left(2 n_{1} n_{2}-n_{1}-n_{2}\right) .
$$


2.3. Composition. The composition $G_{1}\left[G_{2}\right]$ (also known as the graph lexicographic product) of simple undirected graphs $G_{1}$ and $G_{2}$ is the graph with the vertex set $V\left(G_{1}\left[G_{2}\right]\right)=V_{1} \times V_{2}$ and any two vertices $(x, y)$ and $(u, v)$ are adjacent if and only if $x u \in E_{1}$ or $\left(x=u\right.$ and $\left.y v \in E_{2}\right)$.

Let $G_{1}$ and $G_{2}$ be graphs on $n_{1}>1$ and $n_{2}$ vertices, respectively. It follows from the definition that the distance between two distinct vertices $(x, y)$ and $(u, v)$ of $G_{1}\left[G_{2}\right]$ is given by

$$
d_{G_{1}\left[G_{2}\right]}((x, y),(u, v))= \begin{cases}0, & x=u \text { and } y=v, \\ 1, & x=u \text { and } y v \in E_{2}, \\ 2, & x=u \text { and } y v \notin E_{2}, \\ d_{G_{1}}(x, u), & x \neq u\end{cases}
$$

Note that if $G_{1} \cong K_{1}$ then $G_{1}\left[G_{2}\right] \cong G_{2}$. So, in the following lemma we study the reciprocal complementary Wiener number of composition $G_{1}\left[G_{2}\right]$ for case $n_{1}>1$.

Theorem 2.3. Let $G_{1}$ and $G_{2}$ be two graphs on $n_{1}>1$ and $n_{2}$ vertices, respectively.

(i) If $G_{1}$ is a non-complete graph, then

$$
R C W\left(G_{1}\left[G_{2}\right]\right)=\frac{n_{1} m_{2}}{D_{1}}+\frac{n_{1}\left(\left(\begin{array}{c}
n_{2} \\
2
\end{array}\right)-m_{2}\right)}{D_{1}-1}+n_{2}^{2} R C W\left(G_{1}\right) .
$$

(ii) If $G_{1} \cong K_{n_{1}}$ and $G_{2}$ is a non-complete graph, then

$$
R C W\left(G_{1}\left[G_{2}\right]\right)=\frac{n_{1} m_{2}}{2}+n_{1}\left(\left(\begin{array}{c}
n_{2} \\
2
\end{array}\right)-m_{2}\right)+\frac{n_{2}^{2}}{2}\left(\begin{array}{c}
n_{1} \\
2
\end{array}\right) .
$$

(iii) If $G_{1}$ and $G_{2}$ are complete graphs, then

$$
R C W\left(G_{1}\left[G_{2}\right]\right)=\left(n_{1}+n_{2}^{2}\right)\left(\begin{array}{c}
n_{1} \\
2
\end{array}\right) .
$$

Proof. By the definition of the composition of two graphs one can see that,

$$
D=D\left(G_{1}\left[G_{2}\right]\right)= \begin{cases}1, & G_{1} \cong K_{n_{1}} \text { and } G_{2} \cong K_{n_{2}}, \\ 2, & G_{1} \cong K_{n_{1}} \text { and } G_{2} \nsucceq K_{n_{2}}, \\ D_{1}=D\left(G_{1}\right), & G_{1} \nsubseteq K_{n_{1}} .\end{cases}
$$

Suppose $G_{1}$ and $G_{2}$ are non-complete graphs, then

$$
\begin{aligned}
R C W\left(G_{1}\left[G_{2}\right]\right)= & \sum_{\{(x, y),(u, v)\} \subseteq V\left(G_{1}\left[G_{2}\right]\right)} \frac{1}{D+1-d_{G_{1}\left[G_{2}\right]}((x, y),(u, v))} \\
= & \sum_{\substack{\{(x, y),(x, v)\} \subseteq V\left(G_{1}\left[G_{2}\right]\right) \\
y v \in E_{2}}} \frac{1}{D_{1}}+\sum_{\substack{\{(x, y),(x, v)\} \subseteq V\left(G_{1}\left[G_{2}\right]\right) \\
y v \notin E_{2}}} \frac{1}{D_{1}-1} \\
& +\sum_{\substack{\{(x, y),(u, v)\} \subseteq V\left(G_{1}\left[G_{2}\right]\right) \\
x \neq u}} \frac{1}{D_{1}+1-d_{G_{1}}(x, u)}
\end{aligned}
$$




$$
=\frac{n_{1} m_{2}}{D_{1}}+\frac{n_{1}\left(\left(\begin{array}{c}
n_{2} \\
2
\end{array}\right)-m_{2}\right)}{D_{1}-1}+n_{2}^{2} R C W\left(G_{1}\right),
$$

which completes part (i).

The proof is completed by a similar argument as proof of the first part.

2.4. Disjunction. The disjunction $G_{1} \wedge G_{2}$ of graphs $G_{1}$ and $G_{2}$ is the graph with vertex set $V_{1} \times V_{2}$ and $(x, y)$ is adjacent with $(u, v)$ whenever $x u \in E_{1}$ or $y v \in E_{2}$.

Let $G_{1}$ and $G_{2}$ be graphs on $n_{1}>1$ and $n_{2}>1$ vertices, respectively. Clearly, the distance between two vertices $(x, y)$ and $(u, v)$ of $G_{1} \wedge G_{2}$ is given by

$$
d_{G_{1} \wedge G_{2}}((x, y),(u, v))= \begin{cases}0, & x=u \text { and } y=v, \\ 1, & x u \in E_{1} \text { or } y v \in E_{2}, \\ 2, & \text { otherwise. }\end{cases}
$$

Note that if $n_{i}=1$ for some $i \in\{1,2\}$, then $G_{1} \wedge G_{2} \cong G_{n_{i}^{\prime}}$, where $n_{i}^{\prime}=3-i$. So, we determine the reciprocal complementary Wiener number of disjunction $G_{1} \wedge G_{2}$ for cases $n_{1}>1$ and $n_{2}>1$.

Theorem 2.4. Let $G_{1}$ and $G_{2}$ be graphs on $n_{1}>1$ and $n_{2}>1$ vertices, respectively.

(i) If $G_{1}$ and $G_{2}$ are complete graphs, then

$$
R C W\left(G_{1} \wedge G_{2}\right)=\frac{1}{2}\left[n_{1}\left(\begin{array}{c}
n_{2} \\
2
\end{array}\right)+n_{2}\left(\begin{array}{c}
n_{1} \\
2
\end{array}\right)+\left(\begin{array}{c}
n_{1} \\
2
\end{array}\right)\left(\begin{array}{c}
n_{2} \\
2
\end{array}\right)\right] .
$$

(ii) If $\left\{G_{1}, G_{2}\right\} \neq\left\{K_{n_{1}}, K_{n_{2}}\right\}$, then

$$
R C W\left(G_{1} \wedge G_{2}\right)=\frac{1}{2}\left(n_{1}^{2} n_{2}^{2}+2 m_{1} m_{2}-m_{2} n_{1}^{2}-m_{1} n_{2}^{2}-n_{1} n_{2}\right) .
$$

Proof. From definition of disjunction it is clear that if at least one of graphs $G_{1}$ and $G_{2}$ is not complete, then $D=D\left(G_{1} \wedge G_{2}\right)=2$, otherwise $D=1$. To prove part (ii), assume that $\left\{G_{1}, G_{2}\right\} \neq\left\{K_{n_{1}}, K_{n_{2}}\right\}$. Hence, we can write

$$
\begin{aligned}
R C W\left(G_{1} \wedge G_{2}\right)= & \sum_{\{(x, y),(u, v)\} \subseteq V\left(G_{1} \wedge G_{2}\right)} \frac{1}{D+1-d_{G_{1} \wedge G_{2}}((x, y),(u, v))} \\
= & \sum_{\substack{\{(x, y),(u, v)\} \subseteq V\left(G_{1} \wedge G_{2}\right) \\
x u \in E_{1}}} \frac{1}{2}+\sum_{\substack{\{(x, y),(u, v)\} \subseteq V\left(G_{1} \wedge G_{2}\right) \\
y v \in E_{2}}} \frac{1}{2} \\
& -\sum_{\substack{\{(x, y),(u, v)\} \subseteq V\left(G_{1} \wedge G_{2}\right) \\
x u \in E_{1}, y v \in E_{2}}} \frac{1}{2}+\sum_{\substack{\{(x, y),(x, v)\} \subseteq V\left(G_{1} \wedge G_{2}\right) \\
y v \notin E_{2}}} 1 \\
& +\sum_{\substack{\{(x, y),(u, y)\} \subseteq V\left(G_{1} \wedge G_{2}\right) \\
x u \notin E_{1}}} 1+\sum_{\substack{\{(x, y),(u, v)\} \subseteq V\left(G_{1} \wedge G_{2}\right) \\
x u \notin E_{1}, y v \notin E_{2}}} 1 \\
= & \frac{1}{2}\left(n_{1}^{2} n_{2}^{2}+2 m_{1} m_{2}-m_{2} n_{1}^{2}-m_{1} n_{2}^{2}-n_{1} n_{2}\right) .
\end{aligned}
$$


A similar argument as part (ii), shows that

$$
R C W\left(K_{n_{1}} \wedge K_{n_{2}}\right)=\frac{1}{2}\left[n_{1}\left(\begin{array}{c}
n_{2} \\
2
\end{array}\right)+n_{2}\left(\begin{array}{c}
n_{1} \\
2
\end{array}\right)+\left(\begin{array}{c}
n_{1} \\
2
\end{array}\right)\left(\begin{array}{c}
n_{2} \\
2
\end{array}\right)\right] .
$$

This completes the proof.

2.5. Strong product. The strong product of graphs $G_{1}$ and $G_{2}$, denoted by $G_{1} \otimes G_{2}$, is the graph with vertex set $V_{1} \times V_{2}$ and $(x, y)(u, v)$ is an edge whenever $(x=u$ and $\left.y v \in E_{2}\right)$, or $\left(y=v\right.$ and $\left.x u \in E_{1}\right)$, or $\left(x u \in E_{1}\right.$ and $\left.y v \in E_{2}\right)$.

In the following result, we give a basic property about the strong product of graphs.

Lemma 2.1 ([4]). Let $G_{1}$ and $G_{2}$ be two connected graphs, $x, u \in V\left(G_{1}\right)$ and $y, v \in$ $V\left(G_{2}\right)$. Then $d_{G_{1} \otimes G_{2}}((x, y),(u, v))=\max \left\{d_{G_{1}}(x, u), d_{G_{2}}(y, v)\right\}$.

Corollary 2.4. Let $G_{1} \otimes G_{2}$ be the strong product of connected graphs $G_{1}$ and $G_{2}$. Then $D=\max \left\{D_{1}, D_{2}\right\}$, where $D, D_{1}$ and $D_{2}$ are the diameter of $G_{1} \otimes G_{2}, G_{1}$ and $G_{2}$, respectively.

Theorem 2.5. Let $G_{1} \otimes G_{2}$ be the strong product of connected graphs $G_{1}$ and $G_{2}$. Then

$$
\begin{aligned}
R C W\left(G_{1} \otimes G_{2}\right) \leq & \frac{1}{D}\left(2 m_{1} m_{2}+n_{1} m_{2}+n_{2} m_{1}\right)+\left(n_{1}+2 m_{1}\right)\left(R C W\left(G_{2}\right)-\frac{m_{2}}{D_{2}}\right) \\
& +\left(n_{2}+2 m_{2}\right)\left(R C W\left(G_{1}\right)-\frac{m_{1}}{D_{1}}\right)+2\left[\left(\begin{array}{c}
n_{1} \\
2
\end{array}\right)-m_{1}\right]\left[\left(\begin{array}{c}
n_{2} \\
2
\end{array}\right)-m_{2}\right] .
\end{aligned}
$$

The equality is satisfied if and only if $G_{1}$ or $G_{2}$ is a complete graph.

Proof.

$$
\begin{aligned}
& R C W\left(G_{1} \otimes G_{2}\right)=\sum_{\{(x, y),(u, v)\} \subseteq V\left(G_{1} \bigotimes G_{2}\right)} \frac{1}{D+1-d_{G_{1} \bigotimes G_{2}}((x, y),(u, v))} \\
& =\sum_{\substack{\{(x, y),(x, v)\} \subseteq V\left(G_{1} \otimes G_{2}\right) \\
y v \in E_{2}}} \frac{1}{D}+\sum_{\substack{\{(x, y),(x, v)\} \subseteq V\left(G_{1} \otimes G_{2}\right) \\
y v \notin E_{2}}} \frac{1}{D+1-d_{G_{2}}(y, v)} \\
& +\sum_{\{(x, y),(u, y)\} \subseteq V\left(G_{1} \otimes G_{2}\right)} \frac{1}{D}+\sum_{\substack{\{(x, y),(u, y)\} \subseteq V\left(G_{1} \otimes G_{1}\right) \\
x u \notin E_{1}}} \frac{1}{D+1-d_{G_{1}}(x, u)} \\
& +\sum_{\substack{\{(x, y),(u, v)\} \subseteq V\left(G_{1} \otimes G_{2}\right) \\
x u \in E_{1}, y v \in E_{2}}} \frac{1}{D}+\sum_{\substack{\{(x, y),(u, v)\} \subseteq V\left(G_{1} \bigotimes G_{2}\right) \\
x u \in E_{1}, y v \notin E_{2}}} \frac{1}{D+1-d_{G_{2}}(y, v)} \\
& +\sum_{\substack{\{(x, y),(u, v)\} \subseteq V\left(G_{1} \otimes G_{2}\right) \\
x u \notin E_{1}, y v \in E_{2}}} \frac{1}{D+1-d_{G_{1}}(x, u)} \\
& +\sum_{\substack{\{(x, y),(u, v)\} \subseteq V\left(G_{1} \otimes G_{2}\right) \\
x u \notin E_{1}, y v \notin E_{2}}} \frac{1}{D+1-\max \left\{d_{G_{1}}(x, u), d_{G_{2}}(y, v)\right\}} .
\end{aligned}
$$


By (1.1), we have

$$
\begin{aligned}
n_{1} R C W\left(G_{2}\right)= & \sum_{\substack{\{(x, y),(x, v)\} \subseteq V\left(G_{1} \otimes G_{2}\right) \\
y v \notin E_{2}}} \frac{1}{D_{2}+1-d_{G_{2}}(y, v)} \\
& +\sum_{\substack{\{(x, y),(x, v)\} \subseteq V\left(G_{1} \otimes G_{2}\right) \\
y v \in E_{2}}} \frac{1}{D_{2}+1-\underbrace{d_{G_{2}}(y, v)}_{1}} \\
= & \sum_{\substack{\{(x, y),(x, v)\} \subseteq V\left(G_{1} \otimes G_{2}\right) \\
y v \notin E_{2}}} \frac{1}{D_{2}+1-d_{G_{2}}(y, v)}+\frac{n_{1} m_{2}}{D_{2}},
\end{aligned}
$$

hence,

$$
\begin{aligned}
\sum_{\substack{\{(x, y),(x, v)\} \subseteq V\left(G_{1} \otimes G_{2}\right) \\
y v \notin E_{2}}} \frac{1}{D+1-d_{G_{2}}(y, v)} & \leq \sum_{\substack{\{(x, y),(x, v)\} \subseteq V\left(G_{1} \otimes G_{2}\right) \\
y v \notin E_{2}}} \frac{1}{D_{2}+1-d_{G_{2}}(y, v)} \\
& =n_{1}\left(R C W\left(G_{2}\right)-\frac{m_{2}}{D_{2}}\right) .
\end{aligned}
$$

Similarly, we can check that

$$
\begin{gathered}
\sum_{\substack{\{(x, y),(u, y)\} \subseteq V\left(G_{1} \otimes G_{2}\right) \\
x u \notin E_{1}}} \frac{1}{D+1-d_{G_{1}}(x, u)} \leq n_{2}\left(R C W\left(G_{1}\right)-\frac{m_{1}}{D_{1}}\right), \\
\sum_{\substack{\{(x, y),(u, v)\} \subseteq V\left(G_{1} \otimes G_{2}\right) \\
x u \in E_{1}, y v \notin E_{2}}} \frac{1}{D+1-d_{G_{2}}(y, v)} \leq 2 m_{1}\left(R C W\left(G_{2}\right)-\frac{m_{2}}{D_{2}}\right), \\
\sum_{\substack{\{(x, y),(u, v)\} \subseteq V\left(G_{1} \otimes G_{2}\right) \\
x u \notin E_{1}, y v \in E_{2}}} \frac{1}{D+1-d_{G_{1}}(x, u)} \leq 2 m_{2}\left(R C W\left(G_{1}\right)-\frac{m_{1}}{D_{1}}\right) .
\end{gathered}
$$

Also, we have

$$
\begin{aligned}
\sum_{\substack{\{(x, y),(u, v)\} \subseteq V\left(G_{1} \otimes G_{2}\right) \\
x u \notin E_{1}, y v \notin E_{2}}} \frac{1}{D+1-\underbrace{\max \left\{d_{G_{1}}(x, u), d_{G_{2}}(y, v)\right\}}_{\leq D}} \leq \sum_{\substack{\{(x, y),(u, v)\} \subseteq V\left(G_{1} \otimes G_{2}\right) \\
x u \notin E_{1}, y v \notin E_{2}}} 1 \\
=2\left[\left(\begin{array}{c}
n_{1} \\
2
\end{array}\right)-m_{1}\right]\left[\left(\begin{array}{c}
n_{2} \\
2
\end{array}\right)-m_{2}\right] .
\end{aligned}
$$

On the other hand, it is easy to see that

$$
\begin{aligned}
& \sum_{\substack{\{(x, y),(x, v)\} \subseteq V\left(G_{1} \otimes G_{2}\right) \\
y v \in E_{2}}} \frac{1}{D}=\frac{n_{1} m_{2}}{D}, \quad \sum_{\substack{\{(x, y),(u, y)\} \subseteq V\left(G_{1} \otimes G_{2}\right) \\
x u \in E_{1}}} \frac{1}{D}=\frac{n_{2} m_{1}}{D}, \\
& \sum_{\substack{\{(x, y),(u, v)\} \subseteq V\left(G_{1} \otimes G_{2}\right) \\
x u \in E_{1}, y v \in E_{2}}} \frac{1}{D}=\frac{2 m_{1} m_{2}}{D} .
\end{aligned}
$$


Therefore,

$$
\begin{aligned}
R C W\left(G_{1} \otimes G_{2}\right) \leq & \frac{1}{D}\left(2 m_{1} m_{2}+n_{1} m_{2}+n_{2} m_{1}\right)+\left(n_{1}+2 m_{1}\right)\left(R C W\left(G_{2}\right)-\frac{m_{2}}{D_{2}}\right) \\
& +\left(n_{2}+2 m_{2}\right)\left(R C W\left(G_{1}\right)-\frac{m_{1}}{D_{1}}\right)+2\left[\left(\begin{array}{c}
n_{1} \\
2
\end{array}\right)-m_{1}\right]\left[\left(\begin{array}{c}
n_{2} \\
2
\end{array}\right)-m_{2}\right] .
\end{aligned}
$$

Using similar arguments as in the proof of Theorem 2.5, one can prove the following result.

Lemma 2.2. Let $K_{r}$ be a complete graph on $r$ vertices and $G$ be a graph with $n$ vertices, $m$ edges and diameter $d$. Then

$$
R C W\left(G \otimes K_{r}\right)=\frac{1}{d}\left[r m+d r^{2}\left(R C W(G)-\frac{m}{d}\right)+(n+2 m)\left(\begin{array}{l}
r \\
2
\end{array}\right)\right] .
$$

Example 2.4. By the definition of the composition and strong product of two graphs one can see that, $G\left[K_{n}\right]=G \otimes K_{n}$. The open fence graph is the composition (or strong product) of path $P_{n}$ and $K_{2}$. So, from Theorem 2.3 (i) (or Lemma 2.2), we have

$$
R C W\left(P_{n}\left[K_{2}\right]\right)=R C W\left(P_{n} \otimes K_{2}\right)=\frac{n}{n-1}+4 n-4, \quad n \geq 3 .
$$

As an application, in the following result, we obtain the reciprocal complementary Wiener number of the closed fence graph $C_{n} \otimes K_{2}$.

Lemma 2.3. Let $C_{n}$ be a cycle graph on $n$ vertices. Then

$$
R C W\left(C_{n} \otimes K_{2}\right)= \begin{cases}4 n \sum_{k=1}^{\frac{n}{2}} \frac{1}{k}-2 n+8, & 2 \mid n, \\ 4 n \sum_{k=1}^{\frac{n-1}{2}} \frac{1}{k}+\frac{2 n}{n-1}, & 2 \nmid n .\end{cases}
$$

Proof. We first obtain the reciprocal complementary Wiener number of a cycle graph $C_{n}$ on $n$ vertices. Regarding the structure of the cycle $C_{n}$, it can easily be concluded that if $n$ is even then $D\left(C_{n}, k\right)=n, k=1,2, \ldots, \frac{n}{2}-1$ and $D\left(C_{n}, \frac{n}{2}\right)=\frac{n}{2}$. On the other hand, if $n$ is odd then $D\left(C_{n}, k\right)=n, k=1,2, \ldots, \frac{n-1}{2}$. Hence, by applying relation (1.3), we have

$$
R C W\left(C_{n}\right)= \begin{cases}-\frac{n}{2}+n \sum_{k=1}^{\frac{n}{2}} \frac{1}{k}, & 2 \mid n, \\ n \sum_{k=1}^{\frac{n-1}{2}} \frac{1}{k}, & 2 \nmid n .\end{cases}
$$

Finally, the proof is completed using Lemma 2.2. 
2.6. Symmetric difference. The symmetric difference $G_{1} \oplus G_{2}$ of graphs $G_{1}$ and $G_{2}$ is the graph with vertex set $V_{1} \times V_{2}$ and $(x, y)$ is adjacent with $(u, v)$ whenever $x u \in E_{1}$ or $y v \in E_{2}$ but not both. Note that if $n_{i}=1$ for some $i \in\{1,2\}$, then $G_{1} \oplus G_{2} \cong G_{n_{i}^{\prime}}$, where $n_{i}^{\prime}=3-i$.

In the following lemma, we compute the symmetric difference of two graphs with respect to their numbers of vertices and edges.

Theorem 2.6. Let $G_{1}$ and $G_{2}$ be two graphs on $n_{1}>1$ and $n_{2}>1$ vertices, respectively. Then

$$
R C W\left(G_{1} \oplus G_{2}\right)=\frac{1}{2}\left(4 m_{1} m_{2}+n_{1}^{2} n_{2}^{2}-m_{1} n_{2}^{2}-m_{2} n_{1}^{2}-n_{1} n_{2}\right) .
$$

Proof. By [8, Lemma 4], we have

$$
d_{G_{1} \oplus G_{2}}((x, y),(u, v))= \begin{cases}0, & x=u \text { and } y=v \\ 1, & x u \in E_{1} \text { or } y v \in E_{2}, \text { but not both } \\ 2, & \text { otherwise. }\end{cases}
$$

Hence, by applying these relations, we get $D=D\left(G_{1} \oplus G_{2}\right)=2$. So,

$$
\begin{aligned}
& R C W\left(G_{1} \oplus G_{2}\right)=\sum_{\{(x, y),(u, v)\} \subseteq V\left(G_{1} \oplus G_{2}\right)} \frac{1}{D+1-d_{G_{1} \oplus G_{2}}((x, y),(u, v))} \\
& =\sum_{\substack{\{(x, y),(x, v)\} \subseteq V\left(G_{1} \oplus G_{2}\right) \\
y v \in E_{2}}} \frac{1}{2}+\sum_{\substack{\{(x, y),(x, v)\} \subseteq V\left(G_{1} \oplus G_{2}\right) \\
y v \notin E_{2}}} 1 \\
& +\sum_{\{(x, y),(u, y)\} \subseteq V\left(G_{1} \oplus G_{2}\right)} \frac{1}{2}+\sum_{\substack{\{(x, y),(u, y)\} \subseteq V\left(G_{1} \oplus G_{2}\right) \\
x u \notin E_{1}}} 1 \\
& +\sum_{\substack{\{(x, y),(u, v)\} \subseteq V\left(G_{1} \oplus G_{2}\right) \\
x u \in E_{1}, y v \notin E_{2}}} \frac{1}{2}+\sum_{\substack{\{(x, y),(u, v)\} \subseteq V\left(G_{1} \oplus G_{2}\right) \\
x u \in E_{1}, y v \in E_{2}}} 1 \\
& +\sum_{\substack{\{(x, y),(u, v)\} \subseteq V\left(G_{1} \oplus G_{2}\right) \\
x u \notin E_{1}, y v \in E_{2}}} \frac{1}{2}+\sum_{\substack{\{(x, y),(u, v)\} \subseteq V\left(G_{1} \oplus G_{2}\right) \\
x u \notin E_{1}, y v \notin E_{2}}} 1 \\
& =\frac{1}{2}\left(4 m_{1} m_{2}+n_{1}^{2} n_{2}^{2}-m_{1} n_{2}^{2}-m_{2} n_{1}^{2}-n_{1} n_{2}\right) \text {. }
\end{aligned}
$$

2.7. Corona product. Let $V_{1}=\left\{u_{1}, u_{2}, \ldots, u_{n_{1}}\right\}$ and $V_{2}=\left\{v_{1}, v_{2}, \ldots, v_{n_{2}}\right\}$ be the vertex sets of given graphs $G_{1}$ and $G_{2}$, respectively. The corona product of $G_{1}$ and $G_{2}$ is denoted by $G_{1} \circ G_{2}$ and defined as the graph obtained by taking $n_{1}$ copies of $G_{2}$ and joining each vertex of the $i^{\text {th }}$ copy with vertex $u_{i}$ of $V_{1}, i=1,2, \ldots, n_{1}$. Denote by $G_{2}^{i}$ the $i^{\text {th }}$ copy of $G_{2}$ joined to the vertex $u_{i}$ of $G_{1}$, and let $V_{2}^{i}=\left\{v_{i 1}, v_{i 2}, \ldots, v_{i n_{2}}\right\}$, $i=1,2, \ldots, n_{1}$. 
Theorem 2.7. Let $G_{1}$ and $G_{2}$ be two graphs on $n_{1}>1$ and $n_{2} \geq 1$ vertices, respectively. Then

$$
R C W\left(G_{1} \circ G_{2}\right)<\left(n_{2}+1\right)^{2} R C W\left(G_{1}\right)+\frac{n_{1} n_{2}}{D_{1}+2}+\frac{n_{1}\left[\left(\begin{array}{c}
n_{2} \\
2
\end{array}\right)-m_{2}\right]}{D_{1}+1} .
$$

Proof. From definition of the corona product of graphs, it is easy to check that

$$
\begin{aligned}
d_{G_{1} \circ G_{2}}\left(u_{i}, u_{p}\right) & =d_{G_{1}}\left(u_{i}, u_{p}\right), \\
d_{G_{1} \circ G_{2}}\left(u_{i}, v_{p q}\right) & =d_{G_{1}}\left(u_{i}, u_{p}\right)+1, \\
d_{G_{1} \circ G_{2}}\left(v_{i j}, v_{p q}\right) & = \begin{cases}0, & i=p \text { and } j=q, \\
1, & i=p \text { and } v_{j} v_{q} \in E_{2}, \\
2, & i=p \text { and } v_{j} v_{q} \notin E_{2}, \\
d_{G_{1}}\left(u_{i}, u_{p}\right)+2, & i \neq p .\end{cases}
\end{aligned}
$$

So, we can see that $D=D\left(G_{1} \circ G_{2}\right)=D_{1}+2$. Hence,

$$
\begin{aligned}
R C W\left(G_{1} \circ G_{2}\right)= & \sum_{\{x, y\} \subseteq V\left(G_{1} \circ G_{2}\right)} \frac{1}{D+1-d_{G_{1} \circ G_{2}}(x, y)} \\
= & \sum_{\{x, y\} \subseteq V_{1}} \frac{1}{D+1-d_{G_{1} \circ G_{2}}(x, y)} \\
& +\sum_{i=1}^{n_{1}} \sum_{\left\{v_{i j}, v_{i q}\right\} \subseteq V_{2}^{i}} \frac{1}{D+1-d_{G_{1} \circ G_{2}}\left(v_{i j}, v_{i q}\right)} \\
& +\sum_{i=1}^{n_{1}} \sum_{p=1}^{n_{1}} \sum_{q=1}^{n_{2}} \frac{1}{D+1-d_{G_{1} \circ G_{2}}\left(u_{i}, v_{p q}\right)} \\
& +\sum_{i=1}^{n_{1}-1} \sum_{p=i+1}^{n_{1}} \sum_{j=1}^{n_{2}} \sum_{q=1}^{n_{2}} \frac{1}{D+1-d_{G_{1} \circ G_{2}}\left(v_{i j}, v_{p q}\right)} .
\end{aligned}
$$

Consider now for convenience:

$$
\begin{aligned}
S_{1} & =\sum_{\{x, y\} \subseteq V_{1}} \frac{1}{D+1-d_{G_{1} \circ G_{2}}(x, y)}, \\
S_{2} & =\sum_{i=1}^{n_{1}} \sum_{\left\{v_{i j}, v_{i q}\right\} \subseteq V_{2}^{i}} \frac{1}{D+1-d_{G_{1} \circ G_{2}}\left(v_{i j}, v_{i q}\right)}, \\
S_{3} & =\sum_{i=1}^{n_{1}} \sum_{p=1}^{n_{1}} \sum_{q=1}^{n_{2}} \frac{1}{D+1-d_{G_{1} \circ G_{2}}\left(u_{i}, v_{p q}\right)}, \\
S_{4} & =\sum_{i=1}^{n_{1}-1} \sum_{p=i+1}^{n_{1}} \sum_{j=1}^{n_{2}} \sum_{q=1}^{n_{2}} \frac{1}{D+1-d_{G_{1} \circ G_{2}}\left(v_{i j}, v_{p q}\right)} .
\end{aligned}
$$


So, we have

$$
\begin{aligned}
& S_{1}=\sum_{\{x, y\} \subseteq V_{1}} \frac{1}{D+1-d_{G_{1} \circ G_{2}}(x, y)} \\
& =\sum_{\{x, y\} \subseteq V_{1}} \frac{1}{D_{1}+3-d_{G_{1}}(x, y)} \\
& <R C W\left(G_{1}\right) \text {, } \\
& S_{2}=\sum_{i=1}^{n_{1}} \sum_{\left\{v_{i j}, v_{i q}\right\} \subseteq V_{2}^{i}} \frac{1}{D+1-d_{G_{1} \circ G_{2}}\left(v_{i j}, v_{i q}\right)} \\
& =\sum_{i=1}^{n_{1}} \sum_{\substack{\left\{v_{i j}, v_{i q}\right\} \subseteq V_{2}^{i} \\
v_{j} v_{q} \in E_{2}}} \frac{1}{D_{1}+2}+\sum_{i=1}^{n_{1}} \sum_{\substack{\left\{v_{i j}, v_{i q}\right\} \subseteq V_{2}^{i} \\
v_{j} v_{q} \notin E_{2}}} \frac{1}{D_{1}+1} \\
& =\frac{n_{1} m_{2}}{D_{1}+2}+\frac{n_{1}\left[\left(\begin{array}{c}
n_{2} \\
2
\end{array}\right)-m_{2}\right]}{D_{1}+1}, \\
& S_{3}=\sum_{i=1}^{n_{1}} \sum_{p=1}^{n_{1}} \sum_{q=1}^{n_{2}} \frac{1}{D+1-d_{G_{1} \circ G_{2}}\left(u_{i}, v_{p q}\right)} \\
& =\sum_{i=1}^{n_{1}} \sum_{q=1}^{n_{2}} \frac{1}{D_{1}+2}+\sum_{i=1}^{n_{1}} \sum_{\substack{p=1 \\
p \neq i}}^{n_{1}} \sum_{q=1}^{n_{2}} \frac{1}{D_{1}+2-d_{G_{1}}\left(u_{i}, u_{p}\right)} \\
& <\frac{n_{1} n_{2}}{D_{1}+2}+2 n_{2} R C W\left(G_{1}\right), \\
& S_{4}=\sum_{i=1}^{n_{1}-1} \sum_{p=i+1}^{n_{1}} \sum_{j=1}^{n_{2}} \sum_{q=1}^{n_{2}} \frac{1}{D+1-d_{G_{1} \circ G_{2}}\left(v_{i j}, v_{p q}\right)} \\
& =\sum_{i=1}^{n_{1}-1} \sum_{p=i+1}^{n_{1}} \sum_{j=1}^{n_{2}} \sum_{q=1}^{n_{2}} \frac{1}{D_{1}+1-d_{G_{1}}\left(u_{i}, u_{p}\right)} \\
& =n_{2}^{2} R C W\left(G_{1}\right) \text {. }
\end{aligned}
$$

Therefore,

$$
R C W\left(G_{1} \circ G_{2}\right)<\left(n_{2}+1\right)^{2} R C W\left(G_{1}\right)+\frac{n_{1} n_{2}}{D_{1}+2}+\frac{n_{1}\left[\left(\begin{array}{c}
n_{2} \\
2
\end{array}\right)-m_{2}\right]}{D_{1}+1} .
$$

2.8. Splice and link. Let $G_{1}$ and $G_{2}$ be two connected graphs with disjoint vertex sets $V_{1}$ and $V_{2}$, respectively. For given vertices $u \in V_{1}$ and $v \in V_{2}$, a splice of $G_{1}$ and $G_{2}$ by vertices $u$ and $v$ is denoted by $\left(G_{1} \cdot G_{2}\right)(u, v)$ and defined by identifying the vertices $u$ and $v$ in the union of $G_{1}$ and $G_{2}$. Also, a link of $G_{1}$ and $G_{2}$ by vertices $u$ and $v$ is denoted by $\left(G_{1} \sim G_{2}\right)(u, v)$ and obtained by joining $u$ and $v$ by an edge in the union of these graphs.

Theorem 2.8. Let $G_{1}$ and $G_{2}$ be two graphs on $n_{1}$ and $n_{2}$ vertices, respectively. Then 
(i) $R C W\left(\left(G_{1} \cdot G_{2}\right)(u, v)\right) \leq\left(n_{1}-1\right)\left(n_{2}-1\right)+R C W\left(G_{1}\right)+R C W\left(G_{2}\right)$;

(ii) $R C W\left(\left(G_{1} \sim G_{2}\right)(u, v)\right) \leq n_{1} n_{2}+R C W\left(G_{1}\right)+R C W\left(G_{2}\right)$.

Equality in (i) holds if and only if one of the following cases occurs:

$\left(i_{1}\right) n_{i}=1$, for some $i \in\{1,2\}$;

$\left(i_{2}\right) G_{1}$ and $G_{2}$ are non-complete graphs and $\varepsilon_{G_{1}}(u)=\varepsilon_{G_{2}}(v)=1$.

Moreover, equality in (ii) holds if and only if $n_{1}=n_{2}=1$.

Proof. Suppose $\dot{D}$ and $\tilde{D}$ are the diameter of the splice and link of graphs $G_{1}$ and $G_{2}$ by vertices $u$ and $v$, respectively. By above definitions of the splice and link of graphs, one can easily see that

$$
d_{\left(G_{1} . G_{2}\right)(u, v)}(x, y)= \begin{cases}d_{G_{1}}(x, y), & x, y \in V_{1}, \\ d_{G_{2}}(x, y), & x, y \in V_{2}, \\ d_{G_{1}}(x, u)+d_{G_{2}}(y, v), & x \in V_{1} \text { and } y \in V_{2},\end{cases}
$$

and also,

$$
d_{\left(G_{1} \sim G_{2}\right)(u, v)}(x, y)= \begin{cases}d_{G_{1}}(x, y), & x, y \in V_{1}, \\ d_{G_{2}}(x, y), & x, y \in V_{2}, \\ d_{G_{1}}(x, u)+d_{G_{2}}(y, v)+1, & x \in V_{1} \text { and } y \in V_{2} .\end{cases}
$$

Hence, in graph $\left(G_{1} \cdot G_{2}\right)(u, v)$, if the endpoints of a diametral path (i.e. a shortest path between two vertices whose distance is equal to the diameter of the graph) are in the graph $G_{1}$ (or $G_{2}$ ) then $\dot{D}=D_{1}$ (or $D_{2}$ ), otherwise if one of these endpoints belongs to $V_{1}$ and the other endpoint belongs to $V_{2}$, then $\dot{D}=\varepsilon_{G_{1}}(u)+\varepsilon_{G_{2}}(v)$. Thus, $\dot{D}=\max \left\{D_{1}, D_{2}, \varepsilon_{G_{1}}(u)+\varepsilon_{G_{2}}(v)\right\}$. Similarly, $\tilde{D}=\max \left\{D_{1}, D_{2}, \varepsilon_{G_{1}}(u)+\varepsilon_{G_{2}}(v)+1\right\}$. By applying the above obtained relationships and also definitions of the splice and link of graphs, it is obvious that if $n_{1}=1$ or $n_{2}=1$, then the equality in (i) holds. Assume that $n_{1}, n_{2} \geq 2$, then

$$
\begin{aligned}
& R C W\left(\left(G_{1} \cdot G_{2}\right)(u, v)\right)=\sum_{\{x, y\} \subseteq V\left(\left(G_{1} \cdot G_{2}\right)(u, v)\right.} \frac{1}{\dot{D}+1-d_{\left(G_{1} \cdot G_{2}\right)(u, v)}(x, y)} \\
& =\sum_{\{x, y\} \subseteq V_{1}} \frac{1}{\dot{D}+1-d_{G_{1}}(x, y)}+\sum_{\{x, y\} \subseteq V_{2}} \frac{1}{\dot{D}+1-d_{G_{2}}(x, y)} \\
& +\sum_{\substack{x \in V_{1} \backslash\{u\} \\
y \in V_{2} \backslash\{v\}}} \frac{1}{\dot{D}+1-d_{G_{1}}(x, u)-d_{G_{2}}(y, v)} \\
& \leq\left(n_{1}-1\right)\left(n_{2}-1\right)+R C W\left(G_{1}\right)+R C W\left(G_{2}\right) \text {, }
\end{aligned}
$$

and equality holds when $\dot{D}=D_{1}=D_{2}=d_{G_{1}}(x, u)+d_{G_{2}}(y, v)$, for all $x \in V_{1} \backslash\{u\}$ and $y \in V_{2} \backslash\{v\}$. On the other hand, since $G_{1}$ and $G_{2}$ are connected graphs, we conclude that equality holds if and only if $d_{G_{1}}(x, u)=d_{G_{2}}(y, v)=1$ and $D_{1}=D_{2}=2$, for all $x \in V_{1} \backslash\{u\}$ and $y \in V_{2} \backslash\{v\}$. This means that $G_{1}$ and $G_{2}$ are non-complete graphs 
and $\varepsilon_{G_{1}}(u)=\varepsilon_{G_{2}}(v)=1$, which completes the proof of part (i). The proof of part (ii) can be completed by using the similar arguments as in the proof of part (i).

\section{REFERENCES}

[1] F. M. Brückler, T. Došlić, A. Graovac and I. Gutman, On a class of distance-based molecular structure descriptors, Chemical Physics Letters 503 (2011), 336-338.

[2] X. Cai and B. Zhou, Reciprocal complementary Wiener numbers of trees, unicyclic graphs and bicyclic graphs, Discrete Appl. Math. 157 (2009), 3046-3054.

[3] A. A. Dobrynin, R. Entringer and I. Gutman, Wiener index of trees: theory and applications, Acta. Appl. Math. 66 (2001), 211-249.

[4] W. Imrich and S. Klavzar, Product Graphs: Structure and Recognition, Wiley, New York, 2000.

[5] O. Ivanciuc, QSAR comparative study of Wiener descriptors for weighted molecular graphs, Journal of Chemical Information and Computer Sciences 40 (2000), 1412-1422.

[6] O. Ivanciuc, T. Ivanciuc and A. T. Balaban, The complementary distance matrix, a new molecular graph metric, Acta Chimica Hungarica - Models in Chemistry 137 (2000), 57-82.

[7] D. Janežič, A. Milićević, S. Nikolić and N. Trinajstić, Graph Theoretical Matrices in Chemistry, University of Kragujevac, Kragujevac, 2007.

[8] M. H. Khalifeh, H. Yousefi-Azari and A. R. Ashrafi, The hyper-Wiener index of graph operations, Comput. Math. Appl. 56 (2008), 1402-1407.

[9] R. Nasiri, H. Yousefi-Azari, M. R. Darafsheh and A. R. Ashrafi, Remarks on the Wiener index of unicyclic graphs, J. Appl. Math. Comput. 41 (2013), 49-59.

[10] H. Ramane and V. Manjalapur, Reciprocal Wiener index and reciprocal complementary Wiener index of line graphs, Indian Journal of Discrete Mathematics 1 (2015), 23-32.

[11] Z. K. Tang and H. Y. Deng, The graphs with minimal and maximal Wiener indice among a class of bicyclic graphs, J. Nat. Sci. Hunan Norm. Univ. 31 (2008), 27-30.

[12] H. Wiener, Structural determination of paraffin boiling points, J. Amer. Chem. Soc. 69 (1947), $17-20$.

[13] K. Xu, M. Liu, K. C. Das, I. Gutman and B. Furtula, A survey on graphs extremal with respect to distance-based topological indices, MATCH Commun. Math. Comput. Chem. 71 (2014), 461-508.

[14] B. Zhou, X. Cai and N. Trinajstić, On reciprocal complementary Wiener number, Discrete Appl. Math. 157 (2009), 1628-1633.

[15] Y. Zhu, F. Wei and F. Li, Reciprocal complementary Wiener numbers of non-caterpillars, Appl. Math. 7 (2016), 219-226.

DEPARTMENT OF MATHEMATICS, Imam Hossein Comprehensive University, TEHRAN, IRAN

Email address: R.Nasiri.82@gmail.com

Email address: kpnakhaei@ihu.ac.ir

Email address: ashojaeifard@ihu.ac.ir 